\title{
Votre prévoyance est-elle optimalisée fiscalement?
} Cinq questions concernant votre prévoyance

Vous êtes certainement agacé du montant de vos impôts? De nombreux consœurs et confrères se sont déjà informés chez nous pour connaître les possibilités d'optimalisation et sont très satisfaits de nos conseils. Sur la base des questions qui nous sont posées nous vous avons résumé les cinq points les plus importants sous forme d'un court test:
Avez-vous épuisé le potentiel de rachat de votre LPP?
OUI
NON

Les rachats de la LPP peuvent être entièrement déduits de votre revenu. Ainsi vous pouvez selon l'importance de votre revenu et du lieu de taxation réaliser une économie d'impôts jusqu'à $45 \%$ avec un rachat de la LPP.
Est-ce que votre fortune est placée d'une manière optimale fiscalement?
$\bigcirc$ OUI
NON

Le gain en capital (p.ex. augmentation des cours en bourse) est libéré fiscalement, alors que le produit du capital (p.ex. intérêts) doit être déclaré comme revenu. Avec les solutions flexibles de prévoyance de FMH Insurance Services, vous pouvez répartir votre fortune globale de telle façon que le produit du capital soit constitué principalement dans la LPP (impôt réduit lors du versement du capital) et non dans votre fortune libre dont le produit du capital est imposé fortement.

Avez-vous vérifié si le montant d'épargne de votre LPP pouvait être augmenté?

$\bigcirc$ OUI $\bigcirc$ NON

Lors d'une augmentation de votre cotisation d'épargne de la LPP, un nouveau potentiel de rachat est créé qui peut être utilisé dans votre optimalisation fiscale. Dans une fondation de prévoyance corporative, la cotisation d'épargne peut être fixée à $25 \%$ du salaire assuré.

Disposez-vous de plusieurs comptes $3^{e}$ pilier a?

OUI

NON

Le versement d'un $3^{e}$ pilier a est imposé d'un impôt en prestations de capital dont le taux d'imposition est souvent progressif. Disposez-vous p.ex. de deux comptes $3^{e}$ pilier a, choisissez deux différentes périodes fiscales pour le versement du capital et vous bénéficierez d'un taux fiscal plus bas, d'où moins d'impôts.

Votre épouse/votre époux est-elle/il affiliée/é à une prévoyance professionnelle?

OUI

NON

Souvent dans la réalité le conjoint travaillant au cabinet ne reçoit pas de salaire pour son activité et ainsi n'est pas affilié à une prévoyance LPP. En versant un salaire, le conjoint peut participer à une prévoyance LPP et en plus souscrire un $3^{\mathrm{e}}$ pilier a.

Evaluation

$0 \times \mathrm{NON}$ : Sincères félicitations, vous avez déjà envisagé une optimalisation de votre prévoyance.

1-5× NON: Vous disposez d'un potentiel d'optimalisation important. Nous vous conseillons de vous adresser rapidement à un spécialiste en prévoyance FMH Insurance Services.

Talon réponse

A envoyer ou faxer au: 0319595010

Prénom / Nom

Adresse

NPA / Lieu

Date de naissance

Téléphone privé / cabinet

Atteignable le plus facilement (heure)

Adresse e-mail

Je désire un conseil personnalisé, téléphonez-moi.

Je m’intéresse à:
O Pilier $3 a$
Caisse de pension LPP
Planification retraite
Optimalisation fiscale
Protection juridique
Responsabilité civile prof. 